\title{
Methyl-CpG binding protein 2, receptors of innate immunity and receptor for advanced glycation end-products in human viral meningoencephalitis
}

\author{
Danuta Maslinska ${ }^{1,2}$, Milena Laure-Kamionowska ${ }^{1}$, Sławomir Maslinski ${ }^{2}$ \\ ${ }^{1}$ Department of Experimental and Clinical Neuropathology, Mossakowski Medical Research Centre, Polish Academy of Sciences, \\ Warsaw, ${ }^{2}$ Department of General and Experimental Pathology, Warsaw Medical University, Warsaw, Poland
}

\begin{abstract}
Inflammation is a normal host defense reaction to infections and tissue injury. In pathology, the process of inflammation is deregulated by various environmental factors, prolonged activation of Toll-like receptors (TLRs), induction of epigenetic machinery or expression of receptors for advanced glycation end-products (RAGE). In the present study, we examined immunoexpression of proteins participating in the above-mentioned mechanisms, in the brain of patients with viral meningoencephalitis. The results showed that depending on the period of the disease, the process of inflammation is deregulated in different ways. In an early period of viral meningoencephalitis, we found numerous so-called microglial nodules which were strongly immunopositive to methyl-CpG protein 2 (MeCP2). This protein is an epigenetic factor important for methylation of DNA; therefore, our results suggest that cells collected in the nodules may participate in modification of the host defense reaction. Moreover, in the early period of viral meningoencephalitis, we found that Purkinje cells of the cerebellum contain TLR3 or TLR9 receptors that can recognize viral pathogens and may activate a self-destructive mechanism in these neurons. In the later (advanced) period of viral meningoencephalitis, despite some of the above observations, RAGE protein was detected in the brain of adult and aging patients. It means that in this period of the disease, the inflammatory process may be deregulated by numerous post-translationally modified proteins that are transported to the brain after binding with activated RAGE. In addition, young patients appeared more susceptible to viral infections than adult and aging patients, because most of them died during the early period of meningoencephalitis.
\end{abstract}

Key words: viral meningoencephalitis, glial nodules, methyl-CpG binding protein 2, Toll-like receptors, receptor for advanced glycation end-products.

\section{Introduction}

The human defense reaction to infections includes activation of innate immunity, inflammation, and acquired immunity [4]. The first line of this rescue strategy relates to the function of innate immunity and pathogen detection. Cells participating in this process recognize pathogens via pattern recognition receptors (PRRs), of which the most well-studied are the Toll-like receptors (TLRs). Toll-like receptors are expressed on the surface of the cells or on the intra- 
cellular vesicles and are stimulated by the presence of pathogen-associated molecular patterns (PAMPs) such as cell membrane components, intracellular wall organelle components, and single or double stranded RNA or DNA fragments $[10,19]$. Following TLR stimulation, second messenger-specific intracellular signaling cascades are activated that result in expression of genes for production of key inflammatory mediators [7] such as cytokines, chemokines or adhesion molecules. The integrated activity and release of all these mediators by innate immunity cells contribute to the normal inflammatory response $[2,14]$ and determine the course and severity of the host response to infection. Thus, the second step of the defense strategy of the organism is inflammation. The main role of inflammation is removal of necrotic cells from the injured tissue and stimulation of the repair processes. However, prolonged activation of innate immunity receptors and production of proinflammatory proteins may deregulate the course of inflammation, leading to chronic diseases. In addition, the course of inflammation may be deregulated by endogenous proteins that undergo non-enzymatic transformations. The structure and function of such proteins are changed. They bind to receptors for advanced glycation end-products (RAGE) and are transported into the inflamed tissue, where they modify the host defense reaction $[5,8]$. Inflammation may also be modified by numerous environmental factors through activation of epigenetic processes that change the expression of genes in host cells. The biological machinery of this system comprises a variety of regulatory proteins including DNA methyltransferases, putative demethylases, methyl-CpG binding proteins such as $\mathrm{MeCP} 2$, histone-modifying enzymes and chromatin remodeling complexes [15]. One of the major epigenetic mechanisms is DNA cytosine methylation, which modulates many cellular events including developmental gene regulation, genome defense, and genomic imprinting [16]. Alterations in DNA methylation have been linked to human diseases such as cancer and several mental retardation disorders, suggesting an important role for DNA methylation in brain function [9]. The above-mentioned intrinsic programs and environmental factors that may modulate the human defense reaction require further study with respect to neuroinflammation such as that induced in the central nervous system (CNS) by viral infection. In the human brain, the defense response to viral infection is almost invariably associated with meningeal inflammation and sometimes with involvement of the spinal cord. Despite the diversity of viral causative agents, the reactions of the CNS are rather stereotyped and display similar morphological features in all forms of encephalitis [17]. However, the morphological changes vary both in the emphasis on one or another type of reaction and in their distribution through the cerebrospinal axis. Histologically, viral meningitis and the meningitic component of viral meningoencephalitis are characterized by sparse inflammatory cell infiltrates in the meninges and in the subarachnoid space. Histologic features of viral encephalitis include perivascular and parenchymal mononuclear cell infiltrates, glial cell reactions, and neuronal degeneration. The characteristic glial reaction to viral infection is formation of focal cell collections of "microglial nodules". The nodules are scattered throughout the brain gray and white matter, either in relation to dying neurons or independently of them. The cause of glial nodule formation and their function in viral infected brains are not well known; therefore, in the present study we paid special attention to such cell collections and their participation in above-mentioned mechanisms suspected to have a modulatory effect on inflammation.

\section{Material and methods}

Brain samples were obtained at autopsy of 24 patients with clinical diagnosis of viral meningoencephalitis. The clinical diagnosis was confirmed by neuropathological examination. Tissue samples were fixed in formalin and embedded in paraffin. For the purpose of the present study, brain paraffin blocks of patients were drawn from the archive (Table I) of the Medical Research Institute, Polish Academy of Sciences, Warsaw, Poland. These blocks contained six different brain regions (cerebellum, brain stem, spinal cord, and cerebral cortex of parietal, frontal and temporal lobes) of each patient and were cut into $5 \mu \mathrm{m}$ thick serial sections. Sections were stained with cresyl violet for routine histological examination, and sections containing glial nodules were selected for immunohistochemical study.

The same procedures were employed to study brain sections of 22 control, age-matched patients who died suddenly because of non-neurological disorders, in whom no brain morphological lesions were found and no neurological symptoms were observed before they died.

The study was carried out according to the national ethics guidelines and legal regulations regarding the use of archival post-mortem material. 
Table I. Groups of infected patients

\begin{tabular}{|lcccc|}
\hline Group of patients & Age & Number & Sex (F/M) & Brains with "glial nodules" \\
\hline Neonates & $2-7$ ms & 7 & $2 / 5$ & $7 / 7$ \\
\hline Children & $1-3$ ys & 6 & $2 / 4$ & $6 / 6$ \\
\hline Teenagers & $11-13$ ys & 5 & $4 / 1$ & $4 / 5$ \\
\hline Adults & $55-57$ ys & 3 & $1 / 2$ & $1 / 3$ \\
\hline Aging & $65-78$ ys & 3 & $2 / 1$ & $0 / 3$ \\
\hline
\end{tabular}

ms - months of life, ys-years of age

\section{Immunohistochemistry}

Immunohistochemistry was performed using the avidin-biotin-peroxidase complex system, according to the manufacturer's recommendations (Vectastain Elite ABC Peroxidase Kit; Vector Laboratories).

Briefly, sections were dewaxed and hydrated through descending alcohols to water. For non-enzymatic antigen retrieval, some sections were heated in $0.01 \mathrm{M}$ sodium citrate buffer $\left(\mathrm{pH} \mathrm{6.0)}\right.$ to $95^{\circ} \mathrm{C}$ and allowed to cool for $20 \mathrm{~min}$ at room temperature and washed with PBS. Then, sections were incubated in methanol/3\% $\mathrm{H}_{2} \mathrm{O}_{2}$ solution for $20 \mathrm{~min}$ to quench endogenous peroxidase. After being washed again in PBS and blocked with solution containing PBS $/ 5 \%$ normal serum of goat, rabbit or mouse for $2 \mathrm{~h}$ at room temperature, sections were incubated overnight at $4^{\circ} \mathrm{C}$ in solutions of primary antibodies (Table II).

Immunoreactions were visualized using biotinylated secondary antibodies and ABComplex/HRP conjugate. All sections were lightly counterstained with Mayer's hematoxylin.

For negative controls, primary antibodies were replaced with an appropriate isotypic normal mouse, rabbit or goat immunoglobulin fraction at matched protein concentration. These were included for the examination of each specimen and consistently produced negative results.

\section{Results}

In brain specimens of all our patients affected by viral meningoencephalitis, the morphological chang-

Table II. Antibodies used in the study

\begin{tabular}{|c|c|c|c|c|c|}
\hline Antigen name & Antigen origin & Antigen domain & Catalogue & Antibody type & Dilution \\
\hline GFAP & human & n-terminus & sc-6171 & polyclonal & $1: 50$ \\
\hline GFAP & human & c-terminus & sc- 6170 & polyclonal & $1: 50$ \\
\hline Ferritin & human & full length & DAKO & polyclonal & $1: 100$ \\
\hline MeCP2 & human & c-terminus & SC-5758 & polyclonal & $1: 50$ \\
\hline MeCP2 & human & $187-486$ aа & sc-20700 & polyclonal & $1: 50$ \\
\hline MeCP2 & human & n-terminus & SC-5755 & polyclonal & $1: 50$ \\
\hline TLR2 & human & n-terminus & SC-8689 & polyclonal & $1: 50$ \\
\hline TLR3 & mouse & extracellular & sc-23323 & polyclonal & $1: 50$ \\
\hline TLR4 & human & c-terminus & SC-8694 & polyclonal & $1: 50$ \\
\hline TLR9 & human & near n-term & sc-13215 & polyclonal & $1: 50$ \\
\hline RAGE & human & n-terminus & sc -8230 & polyclonal & $1: 50$ \\
\hline RAGE & human & c-terminus & SC-8229 & polyclonal & $1: 50$ \\
\hline
\end{tabular}

SC-Santa Cruz, USA

GFAP-glial fibrillary acidic protein, ferritin - marker of microglial cells L, TLR - Toll-like receptor, MeCP2 - methyl-CpG binding protein 2, RAGE - receptors for advanced glycation end-products 
es varied with the stage of the disease at which the patient died. Generally, in the early stage, the brain was infiltrated with small cells, and most of them appear to be lymphocytes and histiocytes, probably of microglial origin. These cells formed numerous focal "stars" or "microglial nodules". The nodules differed in size and cell density from a few up to 100 or more cells per nodule (Fig. 1). All such cell collections were strongly immunoreactive for methyl-CpG binding protein 2 (MeCP2) (Table III). MeCP2 immunopositive nodules were scattered throughout the cerebral gray (Fig. 2) and white matter (Fig. 3). Some nodules were seen in the close vicinity of dying nerve cells, suggesting that MeCP2 immunopositive cells of the nodules participated in neuronal degeneration and/ or neuronophagia (Fig. 4). Corresponding to these lesions, prominent multifocal, MeCP2 immunopositive glial nodules were also observed in the cerebellar cortex (Table III). Generally, glial nodules were most numerous in brains of neonates, infants and teenagers (Table I), suggesting that young patients were more susceptible than adults to viral infection and died in the early period of the disease.

In the cerebellum of all infected patients, innate immunity receptors that recognize viral pathogens such as TLR3 and TLR9 were found. They were detected in the cytoplasm of Purkinje cells and, depending on the virus type which caused infection of the patient, in some patients these cells were immunopositive for TLR3 and in others for TLR9 (Fig. 5).

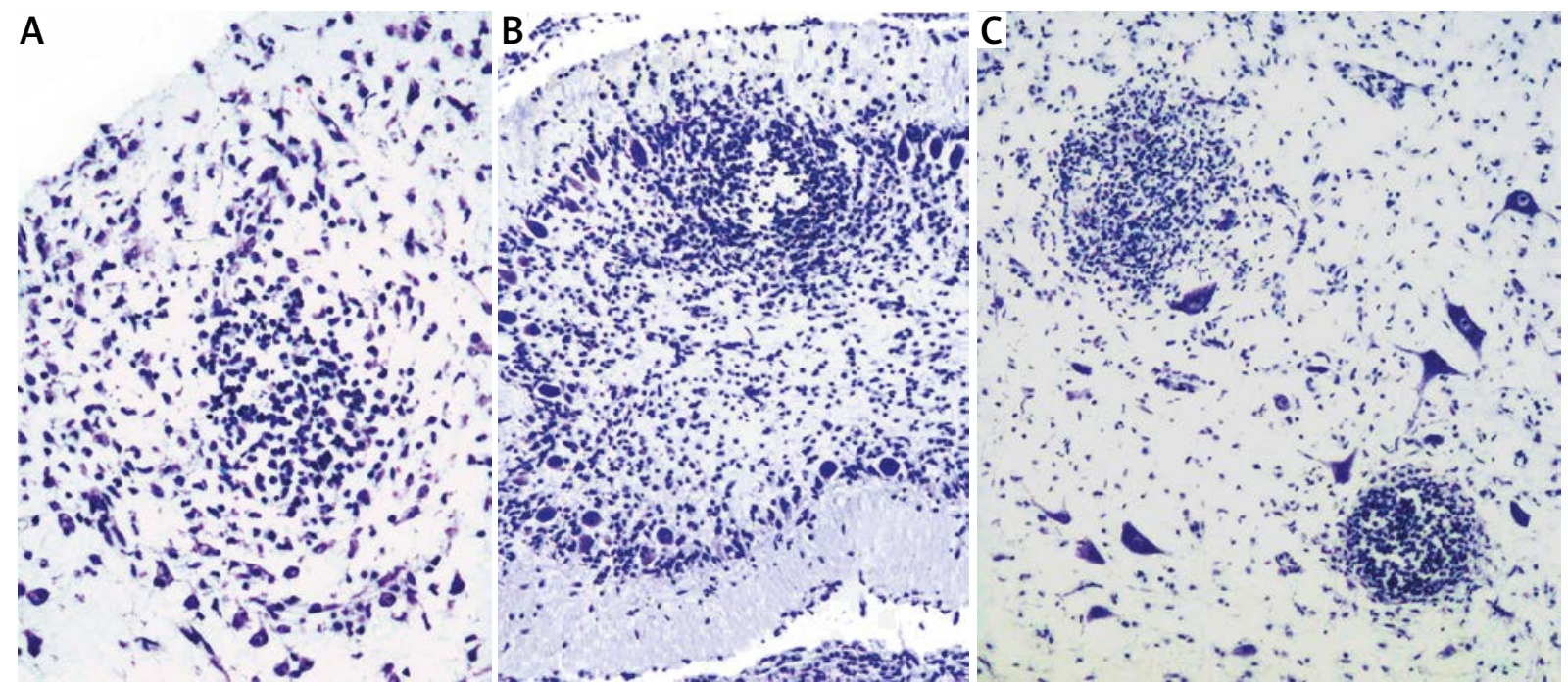

Fig. 1. Different size and cell density of microglial nodules in patients with viral meningoencephalitis (cresyl violet, 500x). A) Microglial nodule in the cerebral cortex gray matter of a 2-year-old patient. B) Microglial nodule in the cortex of the cerebellum of a 2-month-old infant. C) Microglial nodule in the ventral horn of the spinal cord of a 55-year-old patient.

Table III. Antigens in "glial nodules" detected by antibodies used in the study

\begin{tabular}{|c|c|c|c|c|}
\hline \multirow[t]{2}{*}{ Antigens } & \multicolumn{4}{|c|}{ Localization of nodules in the brain } \\
\hline & cerebral cortex & brain white matter & Purkinje cell degeneration & neuronophagia in spinal cord \\
\hline GFAP & - & - & - & - \\
\hline MeCP2 & + & + & + & + \\
\hline TLR2 & - & - & - & - \\
\hline TLR3 & - & - & - & - \\
\hline TLR4 & - & - & - & - \\
\hline TLR9 & - & - & - & - \\
\hline RAGE & - & - & - & - \\
\hline
\end{tabular}



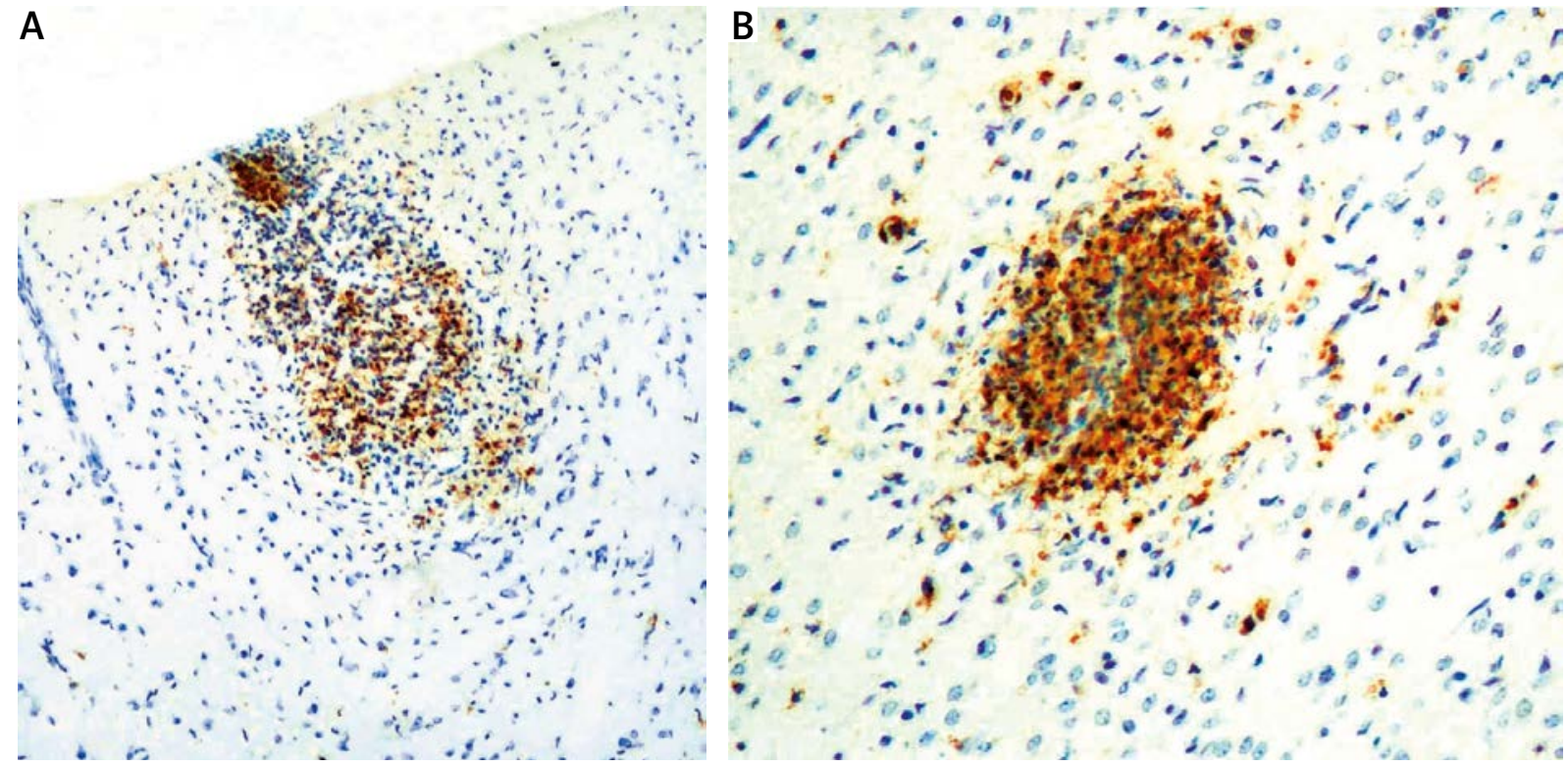

Fig. 2. Methyl-CpG binding protein (MeCP2) detected in immunopositive cells of glial nodules localized in gray matter of the cerebral cortex of patients with viral meningoencephalitis (immunostaining with diaminobenzidine/cresyl violet 350x). A) Nodule in the brain of a 2-year-old patient. B) Nodule in the brain of a 12 -year-old patient.

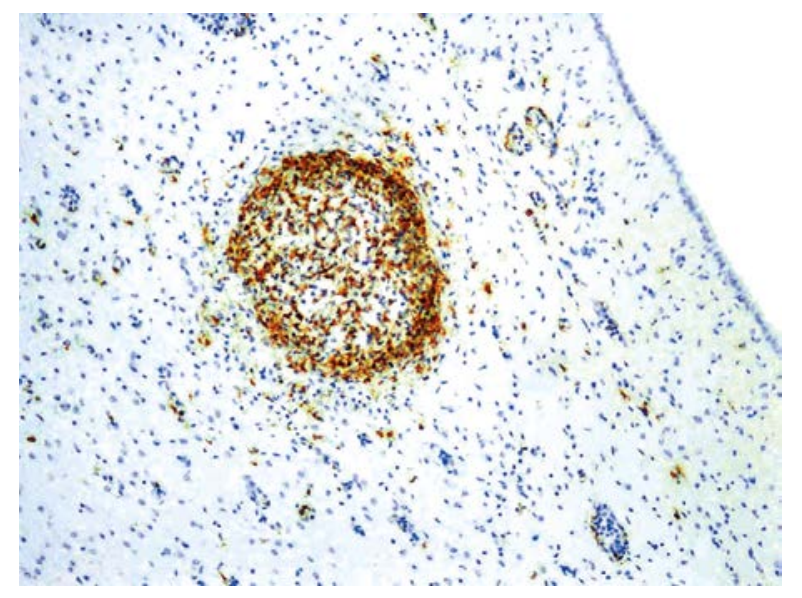

Fig. 3. Methyl-CpG binding protein (MeCP2) detected in immunopositive cells of glial nodules localized in white matter of periventricular area of the brain of a 2-year-old patient with viral meningoencephalitis (immunostaining with diaminobenzidine/cresyl violet 350x).

Such immunoreactivity was observed only in cells with no signs of degeneration, suggesting that in these cells, the process of degeneration could be initiated following recognition of the viral pathogen. In no brain regions were there detected TLR2 and TLR4 receptors that recognize bacterial pathogens. Thus we suspect that bacterial infections were

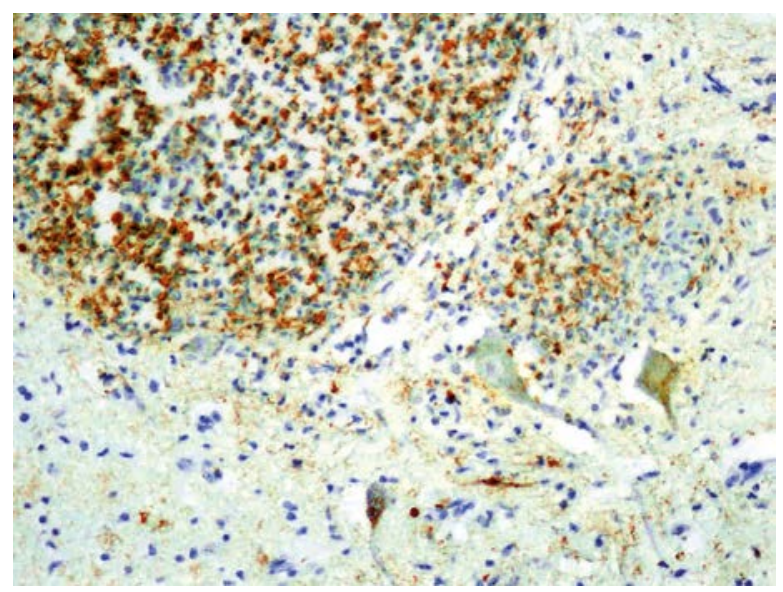

Fig. 4. Methyl-CpG binding protein (MeCP2) detected in immunopositive cells of glial nodules localized in the vicinity of degenerating nerve cells of the ventral horn of the spinal cord in a 55-year-old patient (immunostaining with diaminobenzidine/cresyl violet 500x).

not involved in the process of meningoencephalitis observed in our patients.

In addition, in our adult patients, the inflammatory process could also be modulated by ligands of RAGE receptors, because we found numerous foci of immunoreactivity to RAGE in brain areas with widespread tissue necrosis (Fig. 6A), in hypertrophied gli- 
al cells (Fig. 6B) and in cells localized in the vicinity of blood vessels (Fig. 6C).

\section{Discussion}

Most viral infections of the CNS are potentially serious complications of systemic infections caused by common viral agents. Thus, the initial infection may be acquired through the gastrointestinal tract, respiratory tract, genital tract, percutaneous inoculation, or by transplacental passage. Depending on the particular virus, several pathways are at least potentially available for access to the CNS. Regardless of the route of entry to the CNS, infection with neurotropic viruses activates innate immune responses of macrophages that release proinflammatory cytokines [7]. The brain inflammatory reactions to viral infection tend to be similar in all forms of encephalitis and almost constant are microglial hyperplasia and proliferation [17]. Characteristic for this reaction are also clusters of cells commonly termed "microglial nodules". Microglial nodules are a well-known phenomenon in viral encephalitis, and we observed them most often in the early period of the disease. In the brains of our young patients, nodules were identified in the absence of leukocyte infiltration, tissue necrosis and astrogliosis. In cells of the nodules we found MeCP2 protein, which is an important factor of epigenetic mechanisms. This result suggests that cells of nodules are involved in the process of viral deregulation of the host defense reaction. In previous studies, authors have found MeCP2 to be abundantly expressed in

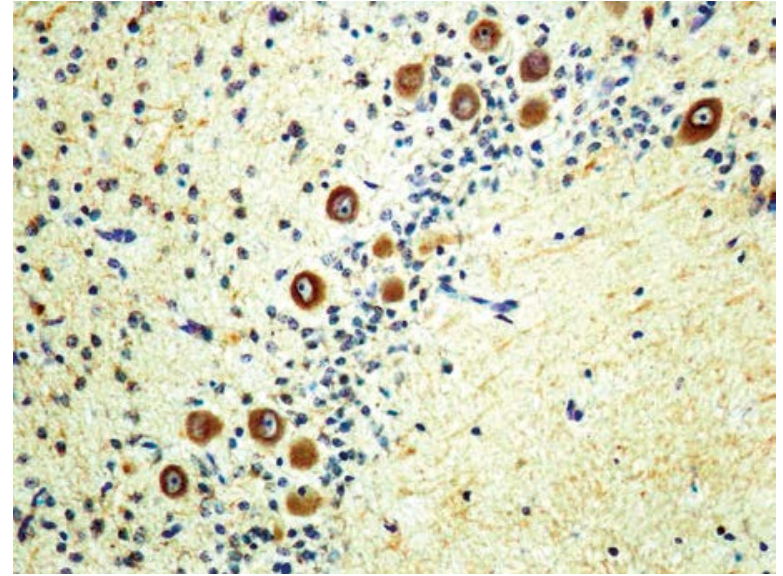

Fig. 5. Immunohistochemical detection of Tolllike receptor 3 (TLR3) protein in cytoplasm of Purkinje cells in the cerebellum of a 2-monthold infant with viral meningoencephalitis (immunostaining with diaminobenzidine/cresyl violet 350x).

neurons and reported that this protein functions as both a transcriptional suppressor and an activator $[3,12,13,20]$. Although information concerning the relationship between viral infection and MeCP2 is limited, several investigations have been reported. Thus, it was found that MeCP2 binds to the latency-associated nuclear antigen (LANA) of Kaposi sarcoma-associated herpesvirus [11], and that MeCP2 was detected in viral infected glial cells [18]; however, the role of MeCP2 in deregulation of the host inflammatory response following viral infection was not considered.
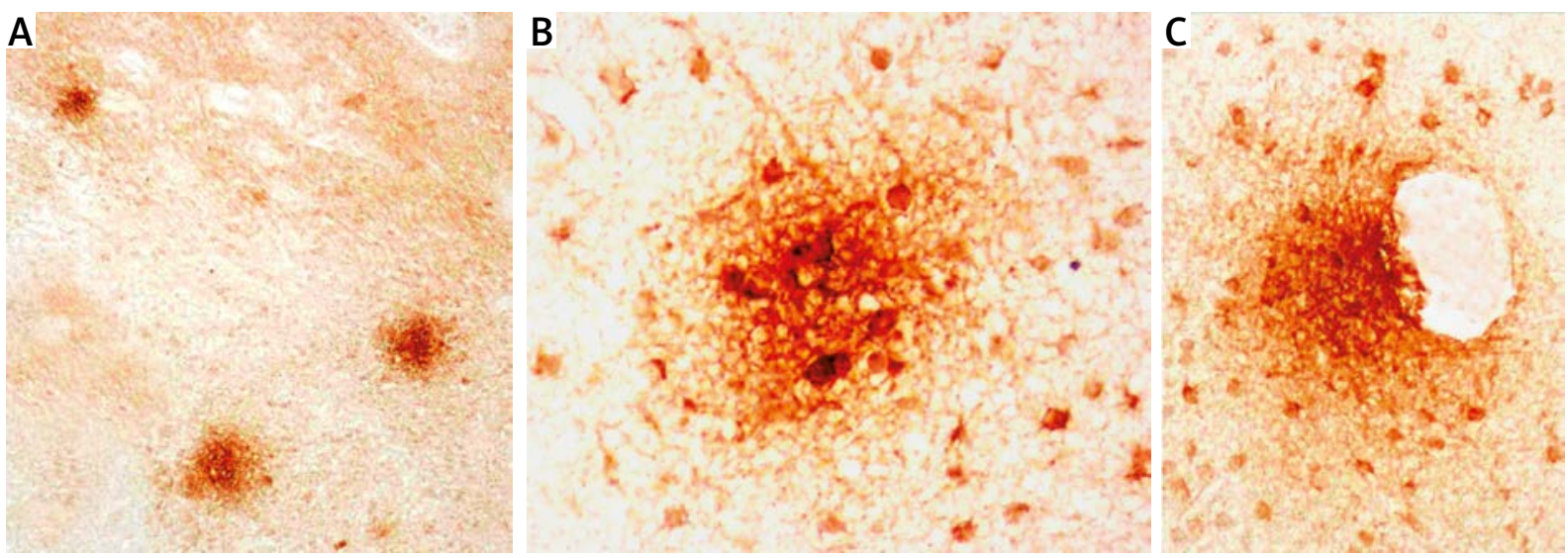

Fig. 6. Immunohistochemical detection of RAGE protein in the brain of a 75-year-old patient with viral meningoencephalitis (immunostaining with diaminobenzidine). A) Focal immunoreactivity to RAGE in white matter of the brain (350x). B) RAGE immunopositive hypertrophied glial cells (500x). C) Perivascular localization of RAGE immunoreactive cells (500x). 
In the later, more advanced period of viral meningoencephalitis, activated microglia can secrete a variety of pro-inflammatory and neurotoxic factors that are necessary to destroy invading pathogens, but these factors can also induce apoptosis of host cells. Additionally, microglia activation is thought to be a causative factor in many neurological diseases, including viral encephalitis [6]. Viruses can injure neurons in a number of ways, including direct killing and induction of apoptosis. Neurotropic viruses can induce neuronal dysfunction of specific neuronal populations, leading to life-threatening consequences for the host [1]. The results of our study suggest that in viral meningoencephalitis, Purkinje cells of the human cerebellum possess one more pathway to neurodegeneration, because we found in their cytoplasm TLR3 or TLR9 receptors that may directly recognize viral pathogens and initiate production of proinflammatory factors.

In the brain of adult and aging patients with viral meningoencephalitis we detected numerous RAGE immunopositive blood vessels and hypertrophied astroglial cells. Specific ligands for this receptor are normal endogenous proteins that spontaneously undergo non-enzymatic transformations, which change their structure and function [5,8]. Accumulation of such proteins, termed advanced glycation end-products (AGE), is characteristic of aging, but accelerated accumulation was observed in different neurological disorders as well. The increased activation of RAGE was observed following oxidative stress, immune and/or inflammatory responses, and upon altered cell functions, suggesting that AGE accumulation also occurs during these processes. Engagement of RAGE introduces transformed proteins into inflamed tissue, modifying the host defense reaction, and induces the release of pro-inflammatory cytokines and free radicals, thus perpetuating a cycle of damage.

In summary, the results of our study suggest that depending on the period of the viral meningoencephalitis, the process of inflammation in CNS may be deregulated by various mechanisms, in which TLR3, TLR9, RAGE and MeCP2 proteins participate. Moreover, for the first time we document that cells collected in glial nodules contain the protein MeCP2, and therefore these cells may play an important but still unknown role in the host defense reaction following viral infection.

\section{Disclosure}

Authors report no conflict of interest.

\section{References}

1. Amor S. Virus infections of the central nervous system. In: Mansons' Tropical Diseases. Cook G, Zumla A (eds.). Sounders Elsevier, London 2008; pp. 853-883.

2. Beutler B. Inferences, questions and possibilities in Toll-like receptor signaling. Nature 2004; 430: 257-263.

3. Chahrour M, Jung SY, Shaw C, Zhou X, Wong ST, Qin J, Zoghbi HY. MeCP2, a key contributor to neurological disease, activates and repress transcription. Science 2008; 320: 1224-1229.

4. Cuenca AG, Wynn JL, Moldawer LL, Levy O. Role of innate immunity in neonatal infection. Am J Perinatol 2013; 30: 105-112.

5. Donato R. RAGE: A single receptor for several ligands and different cellular responses: the case of certain S 100 proteins. Curr Mol Med 2007; 7: 711-724.

6. Ghoshal A, Das S, Ghosh S, Mishra MK, Sharma V, Koli P, Sen E, Basu A. Proinflammatory mediators released by activated microglia induces neuronal death in Japanese encephalitis. Glia 2007; 55: 483-496.

7. Hanke ML, Kielian T. Toll-like receptors in health and disease in the brain: mechanisms and therapeutic potential. Clin Sci (Lond) 2011; 121: 367-387.

8. Hori O, Brett J, Slattery T, Cao R, Zhang J, Chen JX, Nagashima M, Lundh ER, Vijay S, Nitecki D. The receptor for advanced glycation end products (RAGE) is a cellular binding sites for amphoterin. Mediation of neurite outgrowth and co-expression of rage and amphoterin in the developing nervous system. J Biol Chem 1995; 270: 25752-25761.

9. Jaenisch R, Bird A. Epigenetic regulation of gene expression: how the genome integrates intrinsic and environmental signals. Nat Genet 2003; 33 Suppl: 245-254.

10. Kawai T, Akira S. Toll-like receptors and their crosstalk with other innate receptors in infection and immunity. Immunity 2011; 34: 637-650.

11. Krithivas A, Fujimuro M, Weidner M, Young DB, Hayward SD. Protein interactions targeting the latency-associated nuclear antigen of Kaposi's sarcoma-associated herpesvirus to cell chromosomes. J Virol 2002; 76: 11506-11604.

12. Nan X, Campoy FJ, Bird A. MeCP2 is a transcriptional repressor with abundant binding sites in genomic chromatin. Cell 1997; 88: 471-481.

13. Nan X, Ng HH, Johnson CA, Laherty CD, Turner BM, Eisenman RN, Bird A. Transcriptional repression by the methyl-CpG-binding protein MeCP2 involves a histone deacetylase complex. Nature 1998; 393: 386-389.

14. Perry AK, Chen G, Zheng D, Tang H, Cheng G. The host type I interferon response to viral and bacterial infections. Cell Res 2005; 15: 407-442.

15. Robertson KD, Wolffe AP. DNA methylation in health and disease. Nat Rev Genet 2000; 1: 11-19.

16. Rodríguez-Dorantes M, Téllez-Ascencio N, Cerbón MA, López M, Cervantes A. DNA methylation: an epigenetic process of medical importance. Rev Invest Clin 2004; 56: 56-71. 
17. Schochet SS. Infectious Diseases. In: Clinical Neurosciences. Rosenberg RN (ed.). Churchill Livingston, New York, Edinburgh, London, Melbourne 1983; sec III; pp. 195-240.

18. Shirai S, Takahashi K, Kohsaka S, Tsukamoto T, Isogai H, Kudo S, Sawa H, Nagashima K, Tanaka S. High expression of MeCP2 in JC virus-infected cells of progressive multifocal leukoencephalopathy brains. Neuropathology 2011; 31: 38-41.

19. Trinchieri G, Sher A. Cooperation of Toll-like receptor signals in innate immune defence. Nat Rev Immunol 2007; 7: 179-190.

20. Yasui DH, Peddada S, Bieda MC, Vallero RO, Hogart A, Nagarajan RP, Thatcher KN, Farnham PJ, Lasalle JM. Integrated epigenomic analyses of neuronal MeCP2 reveal a role for long-range interaction with active genes. Proc Natl Acad Sci U S A 2007; 104 19416-19421. 